\title{
Los espacios de movilidad de las poblaciones suburbanas de Pekín*
}

\author{
Yanwei Chai \\ Universidad de Pekín. Facultad del Urbanismo y el Medio Ambiente \\ chyw@pku.edu.cn \\ Yue Shen \\ Universidad Normal del Este de China, Shanghái. \\ Centro de Estudios de las Ciudades Modernas \\ shenyue0519@163.com
}

Recibido: septiembre de 2015

Aceptado: enero de 2017

\section{Resumen}

En relación con la rápida suburbanización de las ciudades chinas, la variedad del entorno, los tipos de vivienda y las comunidades sociales que se han formado son cada vez más relevantes. Tomando Pekín como ejemplo, esta investigación ha estudiado su crecimiento espacial durante las últimas décadas, así como el incremento y la modificación de la población, las viviendas, las industrias y las instalaciones comerciales en los suburbios. Las áreas suburbanas entre la cuarta y la sexta carreteras de circunvalación se han convertido en las zonas con mayor desarrollo durante los últimos años. Concretamente, este estudio investigará la movilidad cotidiana en el sector de Shandi-Qinghe, Pekín, basándose en diarios de actividades de sus habitantes y datos de movilidad captados por GPS durante una semana. Se han estudiado los trayectos hacia el trabajo, los espacios donde se hacen las compras y otras acciones de ocio de tres grupos, definidos según el lugar de actividad laboral y residencia. Existen contrastes evidentes en los perfiles socioeconómicos, las rutas de viaje hacia el trabajo y el lugar de compras y de ocio entre esos tres grupos. En suma, el espacio suburbano de Pekín es cada día más complejo y maduro.

Palabras clave: suburbio; espacio material; espacio de movimientos; datos del GPS; Pekín

* Esta investigación se ha realizado gracias a las siguientes fuentes de financiación: Beca (2012BAJ05B04) de los Proyectos Científicos Nacionales del Duodécimo Plan Quinquenal, China (2012BAJ05B00) y Beca Nacional de las Ciencias Naturales para la colaboración con los Investigadores de Hongkong, Macau y los del extranjero, China (41228001). 
Resum. Els espais de mobilitat de les poblacions suburbanes de Pequin

En relació amb la ràpida suburbanització de les ciutats xineses, la varietat de l'entorn, els tipus d'habitatge i les comunitats socials que s'hi han format són cada cop més rellevants. Prenent Pequín com a exemple, aquesta recerca n'ha estudiat el creixement espacial durant les darreres dècades, així com l'increment i la modificació de la població, els habitatges, les indústries i les instal-lacions comercials als suburbis. Les àrees suburbanes entre la quarta $\mathrm{i}$ la sisena carreteres de circumval.lació han esdevingut les zones que s'han desenvolupat més durant els darrers anys. Concretament, aquest estudi investigarà la mobilitat quotidiana al sector de Shandi-Qinghe, Pequín, basant-se en diaris d'activitats dels seus habitants i en dades de mobilitat captades per GPS durant una setmana. S'hi han analitzat els trajectes cap al treball, els espais on fan les compres i altres activitats de lleure de tres grups, definits segons el lloc de residència i d'ocupació laboral. Existeixen contrastos evidents en els perfils socioeconòmics, les rutes de viatge cap a la feina, el lloc on compren i la manera com es distreuen aquests tres grups. En definitiva, l'espai suburbà de Pequín és cada dia més complex i madur.

Paraules clau: nous creixements urbans; espai material; espai de moviments; dades del GPS; Pequín

\section{Résumé. Les espaces de mobilité de la population suburbaine de Pékin}

En ce qui concerne la suburbanisation rapide des villes chinoises, la variété de l'environnement, les types de logement et les communautés sociales qui se sont formés jouent un rôle de plus en plus important. Cette recherche présentera d'abord, comme exemple, le processus d'expansion de Pékin dans les dernières décennies, ainsi que la croissance dans les faubourgs de variables telles que les habitants, les logements, les industries et les installations commerciales. Le territoire entre le quatrième et le sixième périphérique circulaire est la zone avec la plus grande vitesse de développement lors des dernières années. Cette étude portera spécifiquement sur le secteur de Shandi-Qinghe, à Pékin, en se basant sur les activités quotidiennes et les données de mobilité saisis par GPS pendant une semaine, appliquées à un échantillon d'habitants et de banliusards. Ainsi, nous avons étudié les trajets vers le travail, les espaces où se font les achats et les autres activités de loisir de trois groupes, définis selon leurs lieux de résidence et leur travail. Ces trois groupes montrent des contrastes évidents dans les profils socio-économiques, les trajets domicile-travail, et les lieux d'achats et de loisirs. En résumé, l'espace suburbain de Pékin est de plus en plus complexe et élaboré.

Mots-clés: banlieues; espace matériel; espace de mouvements; données GPS; Pékin

\section{Abstract. Daily mobility spaces of Beijing's suburban population}

Due to the rapid suburbanization of Chinese cities, the diversity of the environment, the types of housing and the social communities that have arisen as a consequence matter more and more. Taking Beijing as an example, this paper examines the spatial growth of these cities in recent decades, as well as the changes produced in the population, the housing stock, the industrial sites and the commercial establishments of the suburbs. The suburban area between the fourth and sixth ring roads has developed most in recent years. Daily mobility in the Shandi-Qinghe sector of Beijing is explored based on the daily activities recorded by a sample of the inhabitants themselves and on mobility data captured by GPS for a week. Journeys to work, spaces of shopping and other leisure activities are analysed, comparing three groups defined by their places of residence and work. These three groups 
show significant differences in terms of their socio-economic profiles, travel routes to work, and places of shopping and entertainment. In short, the suburban area of Beijing is becoming increasingly complex and mature.

Keywords: suburb; material space; space of movements; GPS data; Beijing

\title{
Sumario
}

1. Introducción 3. Espacio de movilidad de los habitantes suburbanos pekineses

2. La expansión de los espacios urbanos y el desarrollo de los suburbanos de Pekín

\author{
4. Conclusión \\ Referencias bibliográficas
}

\section{Introducción}

La suburbanización residencial en China se ha visto favorecida por diferentes procesos: el desarrollo de la urbanización, la renovación de los cascos históricos, los grandes proyectos residenciales puestos en marcha por el Gobierno, las viviendas construidas después de las profundas reformas en el campo y en la ciudad, las necesidades de mejorar las condiciones residenciales y la modernización de los ideales de vida de las personas (Feng et al., 2004 y Li et al., 2008). Asimismo, el régimen de uso del suelo urbano, el mandato de renovar las industrias de las ciudades, las mayores exigencias de calidad en el medio ambiente urbano o los requerimientos empresariales para el desarrollo de sus unidades productivas son algunas de las causas que han favorecido la creciente suburbanización de la industria fabril (Zhou y Meng, 1998). Como consecuencia de la suburbanización residencial, la implantación de supermercados y grandes centros comerciales, con precios ventajosos y más superficie, se ha multiplicado en los suburbios, al tiempo que ha surgido espacio de oficinas en forma de office park en metrópolis como Pekín y Shanghai (Chen y Zhang, 2010). Así pues, los espacios suburbanos se enriquecen cada vez más en China e interaccionan con los centros urbanos. En consecuencia, se puede afirmar que las áreas suburbanas ya forman una parte imprescindible del espacio metropolitano.

En América del Norte, la suburbanización del siglo xx se relacionaba sobre todo con los grupos sociales de altos ingresos. Actualmente, en muchas ciudades europeas, los grupos más ricos permanecen en el centro, mientras que los suburbios acogen principalmente a los asalariados. En la mayoría de los países menos desarrollados, los suburbios, al funcionar como áreas con débil control policial, conforman comunidades menos desarrolladas socialmente y dan lugar a tugurios donde se agrupan los inmigrantes campesinos.

Sin embargo, en China, todas esas funciones coexisten dentro del espacio suburbano (Zhou y Logan, 2008). A partir de los años 1980, una profunda 
renovación y restauración del centro motivó que los habitantes se trasladaran al suburbio. La aparición de las villas de lujo y de barrios cerrados (gated communities) favoreció el aumento de propietarios de automóviles; al mismo tiempo, gran cantidad de migrantes se concentraron en la periferia de la ciudad. En consecuencia, los procesos de suburbanización promovidos en las grandes ciudades de China han dado lugar a espacios altamente contrastados desde el punto de vista social, al estar próximos entre sí - aunque segregados - los barrios cerrados, los de reasentamiento y los polígonos de desarrollo (Wei y Yan, 2006). En este espacio suburbano tan heterogéneo, se encuentran comunidades diferenciadas que incluyen a los que se mudaron a esa zona en diferentes épocas procedentes de distintos lugares (ciudadanos emigrantes, campesinos locales urbanizados, inmigrantes de las zonas rurales y forasteros) y que lo hicieron por múltiples causas (mudanza por el trabajo, reasentamiento en viviendas asequibles, reasentamiento por demolición de sus casas, cambio voluntario para mejorar la condición residencial y habitantes estacionales que poseen dos viviendas), además de los que trabajan allí (empleados suburbanos no locales o trabajadores forasteros). Los contrastes entre diferentes espacios suburbanos y la variedad social existente en ellos complican el análisis y exigen que la investigación los entienda como una realidad muy diversa.

Por otra parte, la rápida suburbanización de las ciudades chinas ha provocado una transformación drástica de las formas de organización del área metropolitana. Mientras dominó la economía planificada, las unidades de trabajo acercaban los lugares de vivienda y de ocupación; sin embargo, con la economía de mercado, ambos tipos de espacios se han ido separando, un hecho que se constata con los procesos de suburbanización.

Asimismo, se ha generado una serie de nuevos problemas urbanos. La mayor distancia a otras partes de la ciudad ha incrementado el tiempo de desplazamiento hacia el trabajo por parte de sus residentes, lo que ha disminuido su calidad de vida e, incluso, ha afectado a la división de responsabilidades y a la relación entre los miembros de las familias (Chen y Zhang, 2010). Por lo que respecta a la ciudad, el incremento de los desplazamientos de larga distancia probablemente provoca una mayor congestión del tráfico y agudiza los problemas medioambientales ( $\mathrm{Ma}$ et al., 2011). Si, además, se tiene en cuenta la variedad de los espacios suburbanos (Zhang y Chai, 2011) y la desigual capacidad de los habitantes para moverse, cabe añadir la segregación y la injusticia a los problemas que se pueden ocasionar. Esto se debe, en cierta medida, a la poca atención prestada a las personas en la planificación y la investigación desarrollada sobre los suburbios en China.

El presente estudio, basado en el caso de Pekín, analiza los espacios suburbanos de la capital y su desarrollo, tanto desde el punto de vista espacial como humano, fijándose en variables como la población, las industrias o las instalaciones comerciales, así como los espacios de movilidad cotidiana de la población que vive o trabaja en los suburbios, según la encuesta y los datos de movilidad recogidos durante una semana. 


\section{La expansión de los espacios urbanos y el desarrollo de los suburbanos de Pekín}

Pekín está situado en la Llanura Norte de China y tiene una superficie de 16.410 kilómetros cuadrados. Al final de 2013, contabilizaba 21,15 millones de habitantes, de los cuales el 38\% no había nacido en la ciudad, si bien llevaba viviendo en ella más de medio año. En ese mismo año, el PIB per cápita de toda la ciudad alcanzaba los 93.213 yuanes (11.185 euros de 2013) y se contabilizaban 5,44 millones de automóviles en circulación.

Pekín es una de las primeras ciudades chinas en las que se ha iniciado el proceso de suburbanización, lo cual ha dado origen a uno de los casos más representativos y de los más analizados por las investigaciones académicas. Yixing Zhou señala que la suburbanización que emprendió esta urbe durante la década de 1980 estimuló los estudios sobre el tema en China (Zhou, 1996). Su ubicación en la Llanura Norte ha brindado a Pekín la posibilidad de crecer espacialmente, gracias a la acción conjunta del traslado de las industrias; la construcción de ciudades satélite; las reformas de la tierra y la vivienda, y los requerimientos de expansión de la propia ciudad. En consecuencia, se ha producido una expansión y un desarrollo vertiginosos de los espacios suburbanos durante más de dos décadas, en un movimiento similar al de «extender una torta»: la población, las industrias, las instalaciones de servicios y el tráfico se desarrollan continuamente en estas áreas transformando intensamente los espacios suburbanos, junto con los de toda la metrópoli.

\subsection{La expansión de los espacios urbanos}

Desde el punto de vista espacial, la acelerada suburbanización experimentada por Pekín se ha visto favorecida por la conversión del suelo agrícola suburbano en no agrícola, lo cual ha dado lugar a espacios urbanizados que han ido creciendo hacia las afueras. Por otra parte, la red viaria, compuesta por carreteras radiales y de circunvalación, ha facilitado la aparición a lo largo de las mismas de barrios residenciales que se han ido construyendo de forma concéntrica (Gu, 1999).

Antes de 1949, las construcciones urbanas de Pekín se limitaban al espacio intramuros del casco histórico (la segunda carretera de circunvalación). Tras la fundación de la República Popular, Pekín recuperó su posición como centro político del país y aplicó su objetivo de desarrollo: «convertir la ciudad consumidora en productora». A medida que la sociedad y la economía se desarrollaban, la ciudad se extendía hacia las periferias (Fang et al., 2002). Desde mediados de la década de 1960, el desarrollo se retrasó por motivos políticos y, hasta la década de 1970, aparte de barrios residenciales nacidos junto con las nuevas zonas industriales, el crecimiento se distribuyó entre la segunda y la tercera carreteras de circunvalación (CC). La expansión hacia la periferia de la ciudad fue protagonizada por los "patios de las unidades de trabajo", que aglutinaban en un mismo lugar la casa, la ocupación laboral y los servicios, y eran el principal elemento constitutivo del espacio urbano (Ma y Zhang, 2006). 
Una vez se inició el período de reforma y de apertura en 1978, la urbanización pekinesa entró en una etapa de desarrollo vertiginoso. Entre 1980 y 1995 , casi se triplicó la superficie edificada del núcleo urbano. Con la finalización de la segunda, la tercera y la cuarta CC, la frontera del centro se desplazó hacia el exterior. Cinco años después, en el 2000, el límite de la ciudad había llegado hasta cerca de la quinta CC. Surgieron contrastes importantes entre el norte y el sur. Mientras, al norte de la cuarta CC, la expansión comercial era intensa y la edificación continua crecía hasta el exterior de la quinta CC a lo largo de la autopista Badaling, enlazando barrios gigantes como Huilongguan, Beiyuan, Tiantongyuan, etc., por el contrario, el desarrollo hacia el sur de la tercera CC todavía permanecía muy atrasado.

En la entrada del nuevo milenio, la construcción de infraestructuras urbanas de gran tamaño permitió completar la red viaria con la creación de otras carreteras de circunvalación y ferrocarriles urbanos. Además, han continuado surgiendo nuevos polígonos y grandes barrios residenciales, de modo que las fronteras de la urbe se han ido desplazando progresivamente hacia las afueras. Por lo que respecta a las vías urbanas, en 2001, 2003 y 2009, se terminaron de construir la cuarta, la quinta y la sexta CC, respectivamente. En cuanto al transporte ferroviario metropolitano, se estrenaron, sucesivamente, las líneas de metro números $13,5,8$ y 10, de modo que, a finales de 2013, se contabilizaban 17 líneas y 270 estaciones en total, con lo que se había creado un modelo eminentemente radial. A día de hoy, el metro pekinés ha llegado a convertirse en el mayor del mundo.

Con el objetivo de reducir las fábricas y la población del centro, trasladándolas a las ciudades satélite y a los pueblos pequeños, el Gobierno de Pekín ha planteado una estructura espacial urbana que consiste en «dos ejes, dos zonas con varios centros» y la construcción de 11 ciudades satélite como Tongzhou, Shunyi, Yizhuang, entre otras. De esta manera, las zonas en torno a la ciudad se han vuelto más atractivas para los habitantes de la capital (Chai y Ta, 2009).

\subsection{El desarrollo de los espacios suburbanos}

La construcción de ciudades satélite industriales es la demostración de que la industria ya existía en la periferia de Pekín al inicio de la República Popular. Sin embargo, la suburbanización temprana, simbolizada por la reducción del número total de habitantes del centro a comienzos de la década de 1980 (Zhou, 1996 y Li et al., 2008), afectó en general a los asalariados peor pagados. Se les obligó a mudarse a las afueras por la reforma del régimen de la vivienda y la reconstrucción de los edificios viejos en la urbe.

Por otra parte, ya existía la suburbanización industrial caracterizada por el traslado de las fábricas del centro. En efecto, en un principio, la mudanza y la transformación de las empresas que contaminaban y que molestaban más fue el principal motivo de la suburbanización. Aunque, aparentemente, el sector terciario creció bastante más en los suburbios que en la ciudad, no se ha podido demostrar que se hubiera producido una suburbanización de base comercial. 
Las familias casi no usaban coches particulares y apenas desempeñaron un papel en esa fase de la suburbanización de Pekín (Zhou, 1999, Zhou y Meng, 1998). A partir de 1990, a medida que se fue intensificando la reforma de la tierra y la vivienda, los procesos de suburbanización de Pekín se aceleraron y, además de la población y la industria, se concentraron otros elementos en los espacios suburbanos, que se volvieron cada vez más complejos y maduros.

\subsubsection{El aumento continuo de la población suburbana}

Desde 1990, en Pekín, coexisten la suburbanización activa y la pasiva (la primera está protagonizada por residentes que buscan las áreas periféricas para vivir y la segunda afecta a los antiguos habitantes de los entornos que descubren que sus espacios de vida se han convertido en auténticas periferias de una gran ciudad). La suburbanización demográfica se intensificó y se hizo más rápida desde entonces, ya que los habitantes empezaron a instalarse en las periferias espontáneamente, en busca de la naturaleza y de un espacio residencial más amplio (Feng et al., 2004). A partir del año 2000, la población suburbana de Pekín siguió creciendo: los enormes barrios residenciales y las ciudades satélite en el suburbio se multiplicaron, lo cual atrajo a una gran cantidad de población que se instaló en esas zonas.

En cuanto a la densidad de población en 2010 (figura 1), cabe señalar que el territorio entre la segunda y la cuarta CC se ha convertido en una zona de elevada ocupación, con más de 20.000 habitantes $/ \mathrm{km}^{2}$ en muchas calles del noroeste y más de 10.000 personas en algunos barrios situados al exterior de la quinta CC (como Tiantongyuan y Shangdi-Qinghe). Al observar la tasa de crecimiento demográfico (figura 2), descubrimos que el espacio entre la quinta y la sexta CC es la zona donde el número de habitantes ha crecido más rápidamente durante la última década. Además, en el norte y en el sureste, el

Figura 1. Distribución de la densidad demográfica de Pekín (2010)
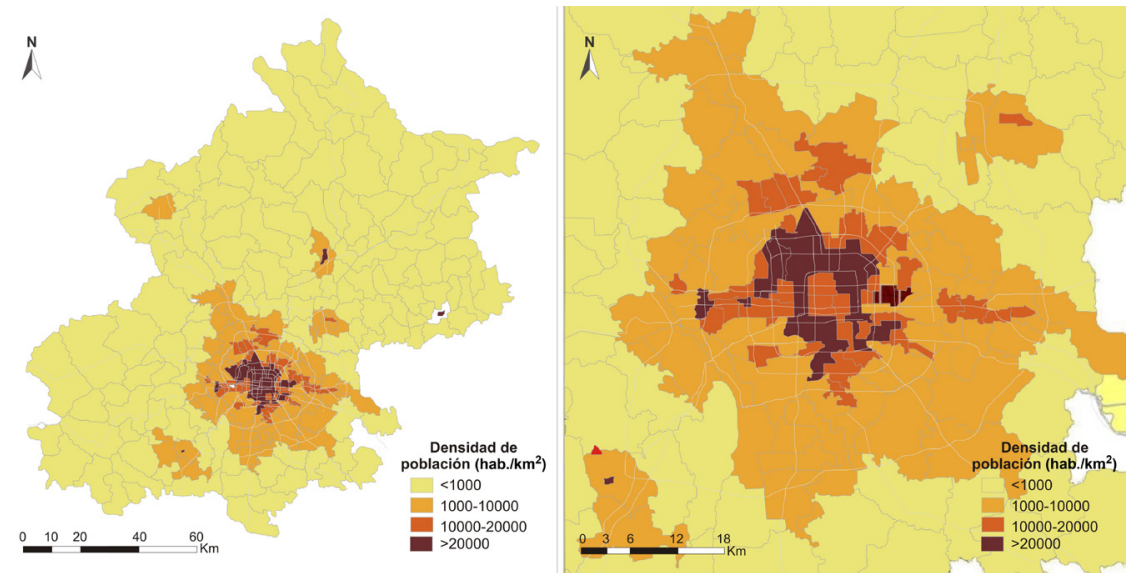

Fuente: el sexto censo de Pekín. 
Figura 2. Tasa del crecimiento demográfico de Pekín (2000-2010)
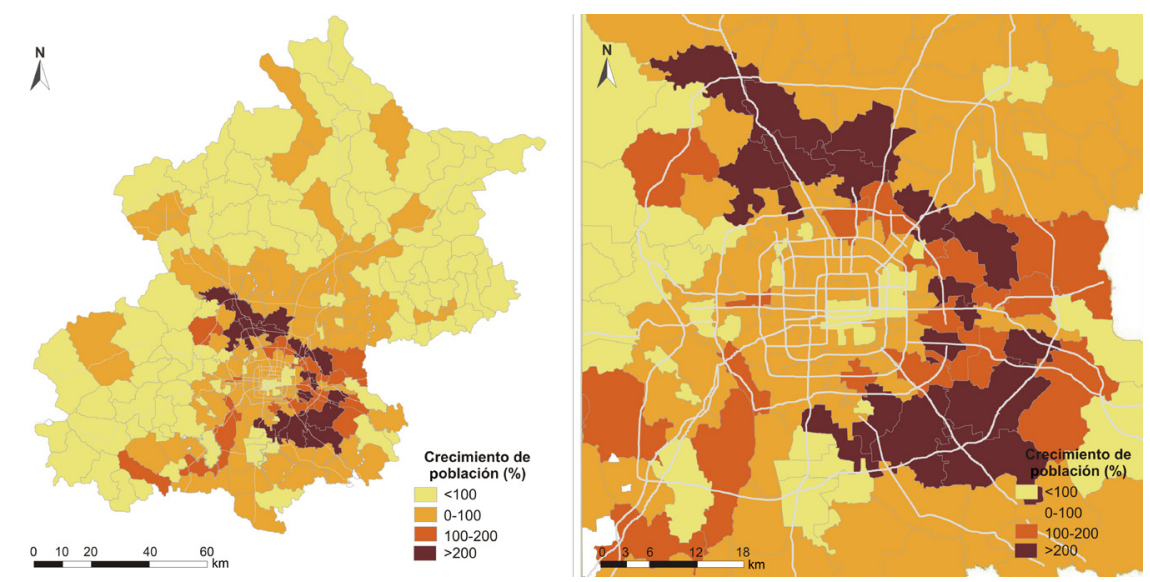

Fuente: el quinto y el sexto censo de Pekín.

incremento se ha producido con mayor velocidad, llegando a ser de un 200\% en barrios como Shangdi, Tiantongyuan, Huilongguan, Yizhuang, etc.

\subsubsection{Con abundantes barrios residenciales construidos en la periferia, el espacio de habitanza se caracteriza por la segregación a nivel micro y la mezcla a nivel macro}

A partir de 1990, la suburbanización residencial impregnó la mentalidad colectiva, debido a que muchos agentes inmobiliarios la impulsaban como una nueva filosofía de vida urbana. Los habitantes eran animados a vivir en los suburbios, atraídos por el reclamo de la baja densidad demográfica. Hasta finales de 1998, se aprobaron los primeros 19 proyectos de viviendas asequibles, siendo Huilonguan, Tiantongyuan y Jiandongyuan los ejemplos más destacados. Después, irían apareciendo barrios residenciales gigantes en los suburbios, especialmente los más periféricos.

La construcción de viviendas de venta libre y asequibles era la forma principal de desarrollar los espacios residenciales suburbanos (Feng et al., 2004; Li et al., 2008). Teniendo en cuenta la distribución de las viviendas construidas después de 1978 y antes de la década de 1990, los barrios residenciales se ubicaban mayoritariamente entre la tercera y la cuarta CC del norte o entre la segunda y la tercera CC del sur, mientras que, después de 1990, se construyeron muchos barrios residenciales enormes cerca de la quinta CC (figura 3) (Wu, 1989; Ma y Zhang, 2006; Yang et al., 2013).

Paralelamente, la división social del espacio según el sector de actividad y la profesión fue perdiendo relevancia, en tanto que el factor económico comenzó a desempeñar un papel importante en la división del espacio residencial. Ello provocó que el precio de las viviendas de venta libre tendiera a disminuir de 
Figura 3. Distribución de los barrios residenciales construidos tras 1978 en Pekín

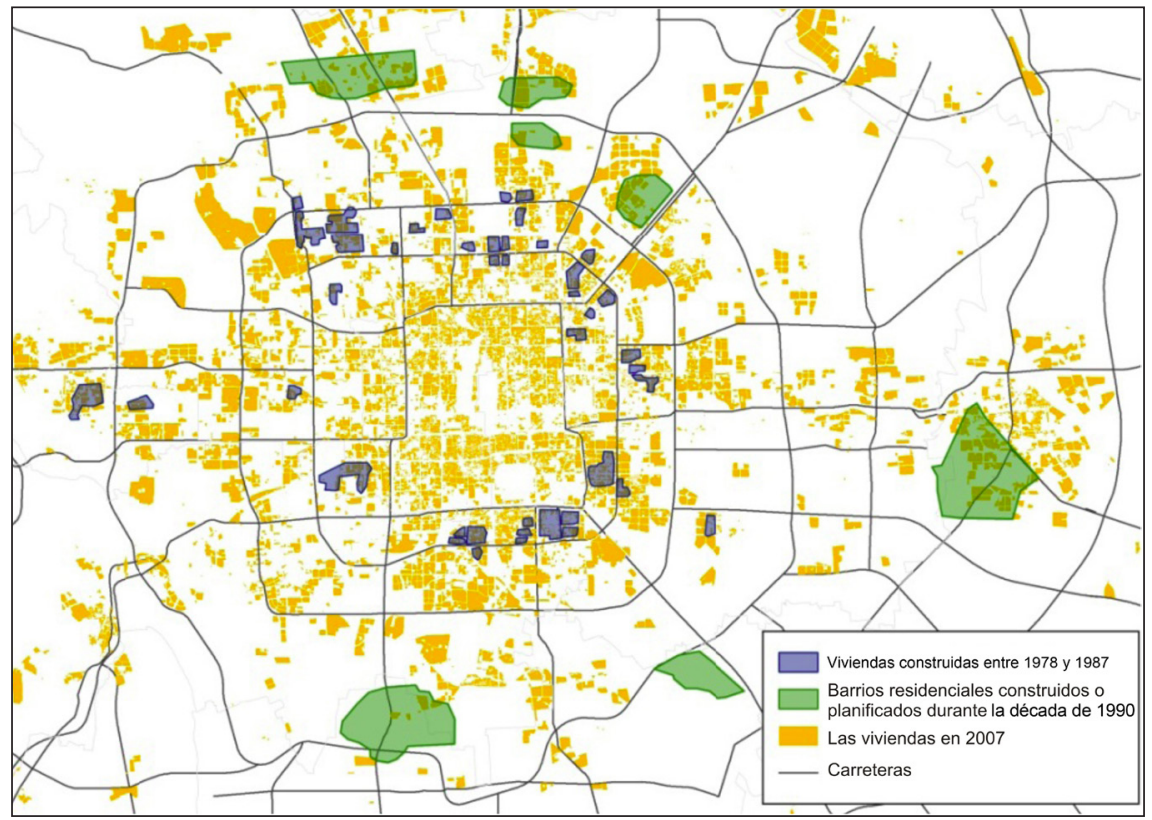

Fuente: Wu, 1989; Yang et al. (2013).

forma concéntrica de centro a periferia, lo que representó la estructura espacial (cabe añadir que el precio del norte era más alto que el del sur).

En contraste con los países occidentales, la división del espacio residencial en Pekín, bajo la influencia combinada de la inercia de la economía planificada y del mecanicismo del mercado, se caracteriza por la segregación a nivel micro y la mezcla a nivel macro. Es decir, el estrato acaudalado se localiza en los barrios suburbanos de villas lujosas y en los pisos de alta calidad en el centro, mientras que las familias con ingresos bajos no tienen otra opción que las viviendas asequibles, baratas, pero con desventajas en referencia al transporte. Por su parte, las comunidades forasteras y las de ingresos bajos están alojadas en los pueblos situados al margen de la zona urbana y en los barrios de chabolas en la ciudad vieja, pendientes de ser reconstruidos (Huang, 2005). Por consiguiente, en los suburbios, las viviendas de venta libre de precios altos, las asequibles y los pueblos periféricos coexisten como espacios residenciales, si bien no cabe duda de que se encuentran segregados entre sí.

\subsubsection{Los polígonos suburbanos han acogido a las fábricas reubicadas y a la industria de tecnología avanzada}

Desde 1990, el Gobierno pekinés ha impulsado el traslado y la transformación de las fábricas contaminantes, obligándolas a mudarse a las afueras. El valor 
alcanzado por el suelo facilitó el reemplazo de la industria pesada por la de bienes de equipo en la zona urbana, lo que se tradujo en un claro impulso de la suburbanización industrial. Las compañías empezaron a salir de la ciudad voluntariamente en busca de mejores condiciones de desarrollo, y los polígonos construidos en la zona suburbana las acogieron, con lo que agruparon la industria fabril en su interior de forma continua (Feng et al., 2004; Zheng y Zhou, 2005). Como el mayor objetivo inicial de los nuevos polígonos pekineses era desarrollar las industrias tecnológicas emergentes, la proporción que ocupaban dichas industrias se incrementaría en el espacio suburbano (Zheng y Qiu, 2005). Este proceso se asemeja al boom de los polígonos en otras partes del país, ya que, después de 1990, también adolecieron de ser demasiado numerosos, estar mal distribuidos y ser ineficaces en el uso del suelo. Tras su reorganización y liquidación, se conservaron tres polígonos nacionales hasta 2011 (que incluían el Polígono de Creación Autónoma de Zhongguancun, el Polígono Económico-Tecnológico de Pekín y la Zona de Servidumbre Sintética de Tianzhu, Pekín) y 16 municipales.

\subsubsection{Los centros comerciales prosperan en el suburbio, mejorando la oferta comercial}

Durante los últimos años, muchos centros comerciales y supermercados gigantes han surgido y han prosperado a gran velocidad en los suburbios de Pekín. Esta dinámica obedece a dos razones principales: por una parte, los supermercados y los grandes almacenes requieren una elevada superficie con arrendamiento relativamente bajo; por ello, la localización en la periferia ayuda a mantener bajos los gastos de funcionamiento. Por otra parte, la suburbanización residencial garantizó la existencia de demanda en el espacio suburbano; además, los precios competitivos de los hipermercados, así como la mejora con-

Figura 4. Densidad de las instalaciones comerciales en 2010 en Pekín
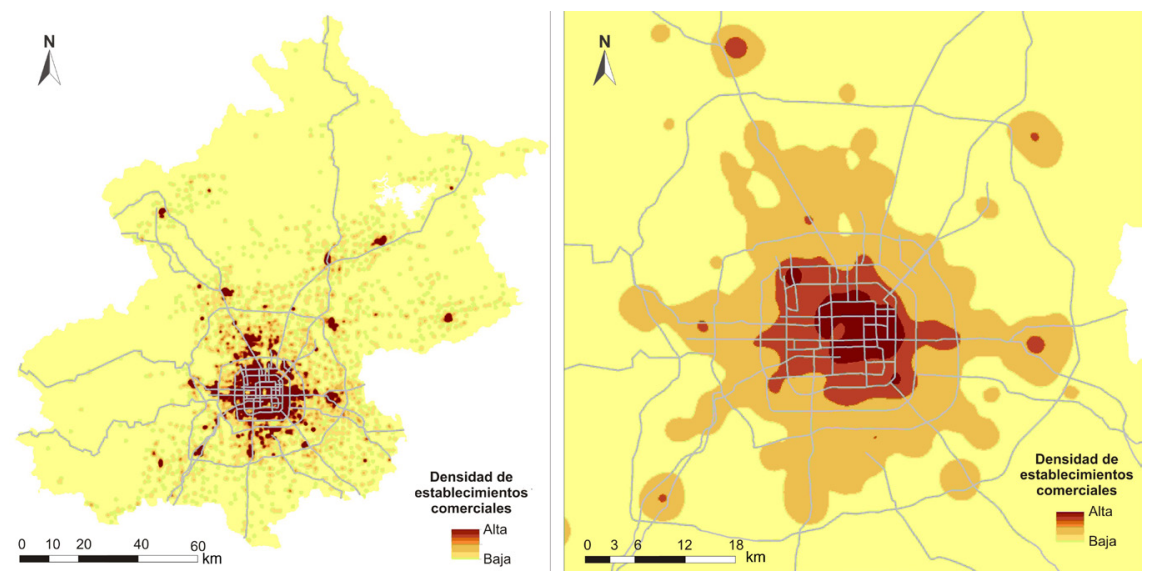

Fuente: datos PDI de las instalaciones comerciales pekinesas. 
tinua de los medios de transporte, han atraído a muchos consumidores (Feng et al., 2004; Long y Chai, 2006). Como consecuencia, han ido apareciendo continuamente supermercados, tiendas y almacenes, entre otras modalidades mercantiles, al tiempo que las instalaciones comerciales vecinas de los barrios residenciales suburbanos han ido mejorando progresivamente. En 2010, la densidad de las instalaciones comerciales de Pekín se caracterizaba, básicamente, por una disminución concéntrica de dentro a fuera. Si, alrededor de la segunda CC, las instalaciones comerciales eran muy densas y las interiores a la cuarta CC, bastante elevadas; entre la cuarta y la sexta CC, dichas instalaciones se localizaban cerca de los barrios residenciales principales (por ejemplo, Qinghe, Yizhuang, etc.), y, fuera de la sexta CC, tan solo se las encontraba en las ciudades satélite o en los pueblos grandes (figura 4).

\section{Espacio de movilidad de los habitantes suburbanos pekineses}

\subsection{El área y los datos}

Después de analizar las características básicas y el proceso de desarrollo del espacio suburbano de Pekín, se llega a la conclusión de que, en estos últimos años, la zona entre la cuarta y la sexta carreteras de circunvalación ha sido el espacio suburbano que más rápidamente se ha desarrollado. En el presente estudio, nos detendremos en el distrito de Shangdi-Qinghe, ubicado entre la quinta y la sexta CC al este del distrito Haidian. Tiene una superficie de $16 \mathrm{~km}^{2}$, está habitado por 240.000 residentes, y 140.000 trabajadores acuden allí cada día. Cuenta con sectores en los que predomina la función residencial (Qinghe) y también con polígonos industriales y tecnológicos (Shangdi).

En relación con estos últimos, alberga tanto polígonos industriales de carácter tradicional como otros emergentes, además de diversos tipos de barrios residenciales construidos en diferentes épocas - como los patios de las unidades de trabajo, las viviendas asequibles, las de venta libre y los pueblos, etc.- - Se trata, por consiguiente, de un sector sintético gigante al noroeste de Pekín, un centro de empleo suburbano y un conjunto residencial importante. Las autopistas y las líneas de metro que atraviesan Shangdi-Qinghe lo conectan con las zonas urbanas vecinas (por ejemplo: Zhongguancun, una región intensiva de empleo, y Huilongguan, un barrio residencial grande, etc.) y con el centro de la ciudad, lo que facilita importantes flujos de movilidad. Por otro lado, una gran parte de los empleados que trabajan en Shangdi-Qinghe no son capaces de comprar su vivienda en dicha zona, mientras que los habitantes de este espacio trabajan en el centro o en otras partes de la ciudad. Esta desconexión entre los espacios de residencia y de trabajo ha agudizado los problemas de tráfico local. Como se trata de una zona de urbanización rápida y de un eje principal de tráfico, constituye un buen modelo para estudiar el espacio suburbano de Pekín.

Los datos del presente análisis provienen de una encuesta sobre las actividades y los desplazamientos cotidianos de los habitantes de Shangdi-Qinghe, Pekín, realizada entre septiembre y diciembre de 2012 por un grupo de investi- 
gadores especializados en la geografía del comportamiento de la Universidad de Pekín. Debido a que esa zona tiene numerosos barrios residenciales y puestos de empleo, el estudio ha realizado un muestreo entre los habitantes de los 23 barrios (exceptuando pueblos y cuarteles), así como entre los empleados de 19 empresas representativas de la Base Industrial de Informática de Shangdi. La investigación se ha llevado a cabo distribuyendo cuestionarios — de los cuales se han obtenido 791 respuestas a través de un sitio web interactivo-y realizando entrevistas en persona o telefónicas con el apoyo de los comités comunitarios y las empresas. Cada caso registra información de una semana e incluye el perfil socioeconómico del entrevistado, el diario de sus actividades durante los siete días y sus trayectorias registradas por GPS. Al final, se han obtenido 709 muestras válidas (que suponen el 89,63\% del total), entre las cuales, 480 corresponden a los barrios residenciales y 229 a las empresas, una distribución que coincide de manera aproximada con las proporciones de los habitantes y los empleados locales. Desde el punto de vista metodológico, se trata de una aproximación tentativa e inicial. No existen precedentes de la realización de encuestas ni entrevistas a habitantes y a usuarios suburbanos. Con un muestreo amplio, y a pesar de las deficiencias que se pueden encontrar en este primer trabajo, el mismo se considera un punto de partida para el análisis del comportamiento y la movilidad urbana en Pekín, una cuestión sin duda novedosa en la literatura geográfica china.

\subsection{Clasificación de personas entrevistadas y sus perfiles socioeconómicos}

Según los casos válidos de la encuesta, una minoría de los residentes de la zona Shangdi-Qinghe trabaja en ella y solo una parte de los empleados de dicha área viven en la misma. A los entrevistados, se les diferencia en tres grupos en función de si su casa y su trabajo están en Shangdi-Qinghe: el primer grupo está formado por los que viven y trabajan en Shangdi-Qinghe; el segundo, por quienes viven allí pero trabajan en otras partes de Pekín, y el tercero reúne a quienes trabajan en la zona pero residen fuera de ella. Por lo tanto, el espacio suburbano de Pekín va a ser estudiado a través del análisis y de la comparación de las características del espacio de movilidad de los tres grupos anteriores.

En base a los datos apuntados por el GPS, se anota dónde viven y trabajan las personas del muestreo. Con el GPS, se busca verificar el nivel de coincidencia de los resultados con los obtenidos en las entrevistas, para poder diseñar en el futuro otros análisis metodológicamente mejor contrastados. De esta manera, tras eliminar aquellos casos cuya información acerca del lugar de su casa y de su trabajo se desconoce, hemos recogido en total 611 casos válidos para el estudio. Los que viven y trabajan en Shangdi-Qinghe suponen un $22,1 \%$ del total; hay 290 personas que viven allí pero que trabajan en otro lugar y que suponen el 47,5\% del total, y 186 empleados en Shangdi-Qinghe que viven fuera, con lo que contribuyen con un 30,4\% al total. Entre todos los habitantes de Shangdi-Qinghe, un 31,8\% trabaja en la zona. Entre ellos, un $42,1 \%$ son habitantes locales. 
Al examinar los perfiles socioeconómicos de los diferentes grupos de personas estudiadas (tabla 1), se aprecia que existen contrastes evidentes. El número de mujeres sobrepasa ligeramente al de hombres. Las personas analizadas tienen un promedio de edad de 35 años y los empleados en esa zona son algo más jóvenes. En relación con el estatus de residencia (bukou), está registrado en la ciudad aproximadamente un $70 \%$ de la muestra. Un 12\% de los residentes en el distrito carecen de residencia registrada en Pekín, mientras que, entre los trabajadores, los no registrados como residentes pekineses alcanzan un $67 \%$. Con respecto al nivel educativo, más de la mitad afirma haber realizado estudios iguales o superiores a licenciatura, por lo que el nivel de educación es alto, especialmente entre los trabajadores que acuden al barrio. Un $75 \%$ del conjunto del muestreo están casados. Entre los que viven en esa zona, un 90\%

Tabla 1. Los perfiles socioeconómicos de los diferentes grupos del suburbio

\begin{tabular}{|c|c|c|c|c|c|c|c|c|c|}
\hline \multirow{2}{*}{\multicolumn{2}{|c|}{$\frac{\text { Categorías }}{\text { Variables categóricas }}$}} & \multicolumn{2}{|c|}{$\begin{array}{l}\text { Viven y trabajan en } \\
\text { Shangdi-Qinghe }\end{array}$} & \multicolumn{2}{|c|}{$\begin{array}{l}\text { Viven en } \\
\text { Shangdi-Qinghe } \\
\text { y trabajan fuera }\end{array}$} & \multicolumn{2}{|c|}{$\begin{array}{l}\text { Trabajan en } \\
\text { Shangdi-Qinghe } \\
\text { y viven fuera }\end{array}$} & \multicolumn{2}{|c|}{ Totalidad } \\
\hline & & $\mathrm{N}$ & Porcentaje & $\mathrm{N}$ & Porcentaje & $\mathrm{N}$ & Porcentaje & $\mathrm{N}$ & Porcentaje \\
\hline Total & & 135 & 100,0 & 290 & 100,0 & 186 & 100,0 & 611 & 100,0 \\
\hline \multirow[t]{2}{*}{ Sexo } & Hombres & 63 & 46,7 & 144 & 49,7 & 77 & 41,4 & 284 & 46,5 \\
\hline & Mujeres & 72 & 53,3 & 146 & 50,3 & 109 & 58,6 & 327 & 53,5 \\
\hline \multirow{2}{*}{$\begin{array}{l}\text { Ciudadanía } \\
\text { pekinesa }\end{array}$} & Sí & 104 & 77,0 & 255 & 87,9 & 61 & 32,8 & 420 & 68,7 \\
\hline & No & 31 & 23,0 & 35 & 12,1 & 125 & 67,2 & 191 & 31,3 \\
\hline \multirow{2}{*}{$\begin{array}{l}\text { Nivel de } \\
\text { educación }\end{array}$} & $<$ Licenciatura & 60 & 44,4 & 144 & 49,7 & 62 & 33,3 & 266 & 43,5 \\
\hline & z Licenciatura & 75 & 55,6 & 146 & 50,3 & 124 & 66,7 & 345 & 56,5 \\
\hline \multirow[t]{2}{*}{ Estado civil } & Solteros & 29 & 21,5 & 28 & 9,7 & 93 & 50,0 & 150 & 24,5 \\
\hline & Casados & 105 & 77,8 & 261 & 90,0 & 92 & 49,5 & 458 & 75,0 \\
\hline \multirow{2}{*}{$\begin{array}{l}\text { Permiso } \\
\text { de conducir }\end{array}$} & Sí & 48 & 35,6 & 136 & 46,9 & 59 & 31,7 & 243 & 39,8 \\
\hline & No & 87 & 64,4 & 154 & 53,1 & 127 & 68,3 & 368 & 60,2 \\
\hline \multirow[t]{2}{*}{ Trabajo } & Estable & 125 & 92,6 & 237 & 81,7 & 180 & 96,8 & 542 & 88,7 \\
\hline & Otros & 10 & 7,4 & 53 & 18,3 & 6 & 3,2 & 69 & 11,3 \\
\hline \multirow{3}{*}{$\begin{array}{l}\text { Nivel de } \\
\text { ingresos }\end{array}$} & Bajo $(<2000)$ & 27 & 20,0 & 54 & 18,6 & 17 & 9,1 & 98 & 16,0 \\
\hline & Mediano (2000-6000) & 86 & 63,7 & 162 & 55,9 & 132 & 71,0 & 380 & 62,2 \\
\hline & Alto (>6000) & 22 & 16,3 & 74 & 25,5 & 37 & 19,9 & 133 & 21,8 \\
\hline \multirow{2}{*}{$\begin{array}{l}\text { Propietario } \\
\text { de viviendas }\end{array}$} & Sí & 87 & 66,4 & 240 & 87,6 & 63 & 35,4 & 390 & 66,9 \\
\hline & No & 44 & 33,6 & 34 & 12,4 & 115 & 64,6 & 193 & 33,1 \\
\hline \multicolumn{2}{|c|}{ Variables continuas } & Promedio & $\begin{array}{l}\text { Desviación } \\
\text { estándar }\end{array}$ & Promedio & $\begin{array}{l}\text { Desviación } \\
\text { estándar }\end{array}$ & Promedio & $\begin{array}{l}\text { Desviación } \\
\text { estándar }\end{array}$ & Promedio & $\begin{array}{l}\text { Desviación } \\
\text { estándar }\end{array}$ \\
\hline \multicolumn{2}{|l|}{ Edad } & 36,49 & 9,54 & 36,87 & 8,81 & 29,40 & 6,97 & 34,51 & 9,11 \\
\hline \multicolumn{2}{|c|}{ Número de familiares } & 2,80 & 1,11 & 3,20 & 1,06 & 2,12 & 1,09 & 2,78 & 1,17 \\
\hline \multicolumn{2}{|c|}{ Número de coches privados } & 0,54 & 0,59 & 0,72 & 0,57 & 0,41 & 0,58 & 0,58 & 0,59 \\
\hline \multicolumn{2}{|c|}{ Duración de la residencia } & 8,82 & 7,27 & 9,91 & 7,74 & 5,61 & 6,71 & 8,35 & 7,56 \\
\hline \multicolumn{2}{|c|}{ Distancia entre casa y trabajo } & 1,42 & 1,03 & 10,42 & 7,13 & 10,19 & 10,28 & 8,12 & 8,44 \\
\hline
\end{tabular}

Fuente: elaboración propia a partir de la encuesta realizada. 
están casados y el número medio de personas de cada núcleo familiar es 3,2 miembros; en cambio, la proporción de los casados entre los trabajadores en esa zona es relativamente baja, con 2,1 personas en cada familia de promedio.

Con respecto a la cantidad de coches privados y la posesión de permisos de conducir, los residentes en esa zona cuentan con más permisos y coches privados que los empleados. Además, los coches en propiedad para los que viven y trabajan en dicha área alcanzan el nivel medio del total, puesto que, al estar las casas cerca de sus trabajos, no les hace falta conducir para ir y volver a los mismos. En este caso, la tasa de posesión de permisos de conducir es relativamente baja.

En cuanto a la profesión y a los ingresos de los encuestados, un 90\% tiene un trabajo fijo. Entre los empleados de esa zona, la mayoría posee un ingreso mediano, mientras que, entre los que viven en ella, predominan los que tienen ingresos altos. Por lo que respecta a la propiedad de la vivienda, un 67\% son propietarios de sus viviendas y la duración de residencia media es de 7,5 años. La tasa de propietarios de viviendas de los empleados es del 35\%. Los que residen allí desde hace menos de 6 años, en promedio, presentan un nivel aparentemente inferior de propiedad a los otros dos grupos.

Se aprecia que, a pesar de la variedad de las viviendas de la zona en cuestión, la mayoría de las mismas son de venta libre y están dirigidas a personas con estudios que residen ahí y trabajan fuera. Los registrados como residentes pekineses, los adinerados, los casados y los propietarios de viviendas forman la mayoría. El Polígono Industrial de TI de Shangdi ofrece numerosos puestos de trabajo y los empleados de la zona que viven fuera tienen una edad relativamente baja y niveles de educación altos. Entre ellos, son mayoría los que no poseen la ciudadanía pekinesa, los solteros, los que no saben conducir y los que alquilan pisos y cuentan con poco tiempo de residencia. Las personas que viven y trabajan en la misma zona presentan un nivel medio, pero, debido a la proximidad entre la casa y el trabajo, solo pocos de ellos cuentan con permiso de conducir.

\subsection{El desplazamiento al trabajo}

Asociando el domicilio particular y el lugar de empleo de cada habitante con el programa ArcGIS, se han analizado las características generales de la movilidad cotidiana por motivos laborales. Se han medido las distancias de casa al trabajo diferenciando grupo por grupo (tabla 1, figuras 5, 6 y 7a). Con el objetivo de mejorar los resultados, se ha realizado un análisis de densidad nuclear (Kernel Density Analysis) sobre el grupo de la muestra que reside en Shangdi-Qinghe y trabaja fuera, así como sobre el que trabaja en la zona de nuestro interés pero reside en otro sitio. Además, vamos a estudiar la dirección lineal (Linear Directional Mean) de los vínculos entre la casa y el trabajo por parte de cada grupo para lograr la dirección en promedio del viaje al trabajo (figura 5, 6b y 7b).

La distancia media de la casa al trabajo que recorren todos los encuestados es de 8,12 kilómetros (en línea recta), si bien un $60 \%$ viaja menos de 10 
Figura 5. Las direcciones de movimiento de las personas que viven y trabajan en ShangdiQinghe

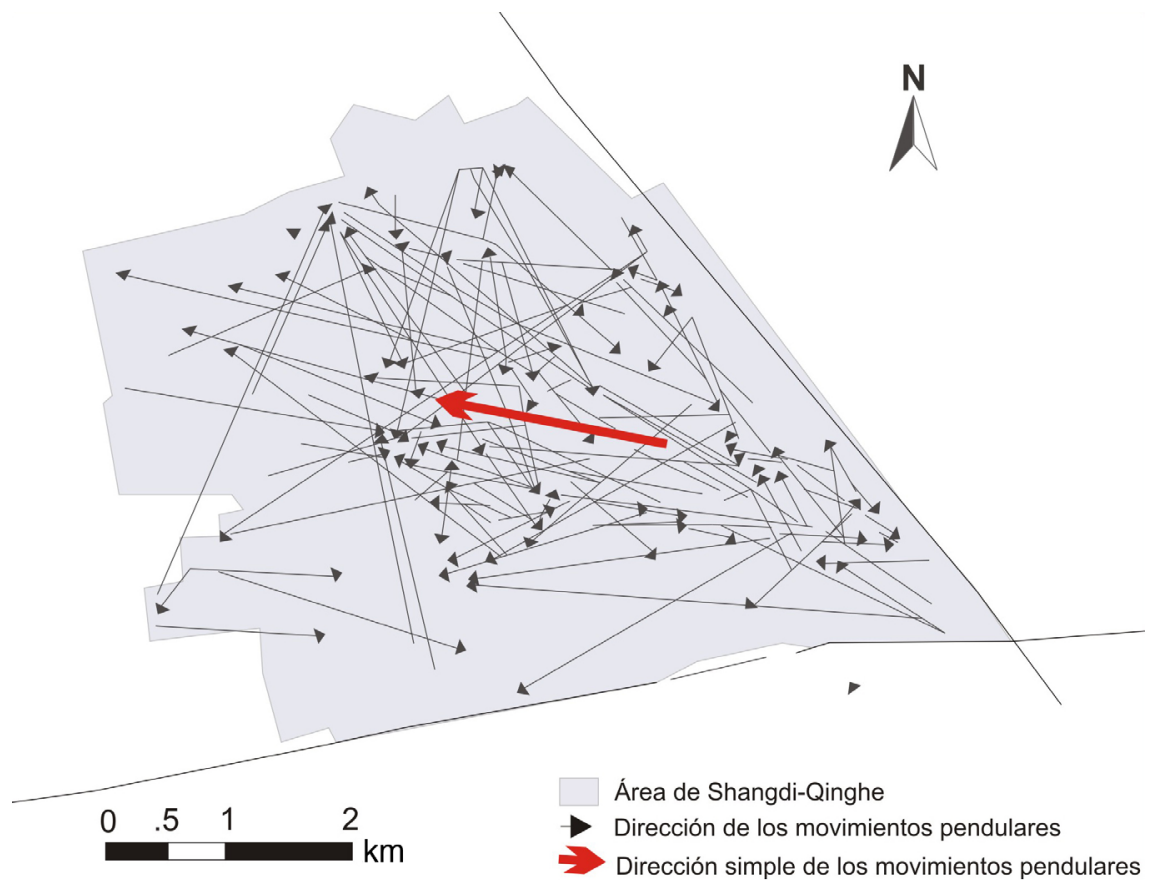

Fuente: elaboración propia a partir de la encuesta realizada.

kilómetros para ir al trabajo, y solo un 10\% tiene que desplazarse a lo largo de más de 20 kilómetros. Los que viven y trabajan en la misma zona solo recorren 1,42 kilómetros de promedio al trabajo en línea recta, lo que es una distancia bastante corta. No obstante, aquellos que habitan en la zona y trabajan fuera, así como los que trabajan en ella pero residen en otro sitio, padecen una distancia de casa al trabajo de aproximadamente 10 kilómetros. De los dos grupos, los residentes locales viajan más, mientras que la desviación estándar del trayecto entre la casa y el lugar de ocupación de los trabajadores es más amplia, es decir, la diferencia de distancias es mayor para estos últimos.

Además, se ha analizado la dirección de los movimientos y las características asociadas. La dirección promedio de la movilidad de los que viven y trabajan en Shangdi-Qinghe es del sureste al noroeste. A través de ese dato, se puede representar la variedad de funciones de los espacios en la zona, ya que hay más barrios residenciales en Qinghe en el este que en Shangdi en el oeste, y los puestos de empleo, excepto los del comercio minorista que se encuentran por todas partes, están concentrados en el polígono industrial de TI de Shangdi al noroeste. Quienes solo viven en Shangdi-Qinghe se mueven generalmente hacia el centro, a la zona de Zhongguancun, y al sur como principales destinos; 
Figura 6. Las características y las direcciones del movimiento de los habitantes locales de Shangdi-Qinghe que trabajan fuera

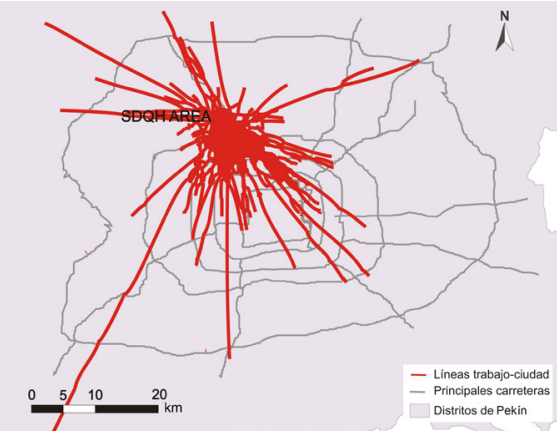

a) Desplazamientos al trabajo por parte de las personas estudiadas.

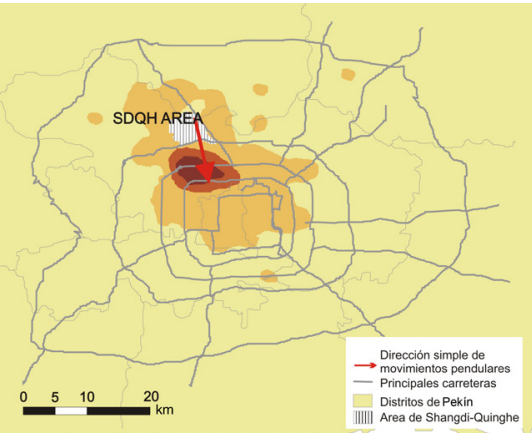

b) Lugar de trabajo y dirección general del movimiento de las personas analizadas.

Fuente: elaboración propia a partir de la encuesta realizada.

Figura 7. Las características y las direcciones del movimiento de los que trabajan en ShangdiQinghe y viven fuera.

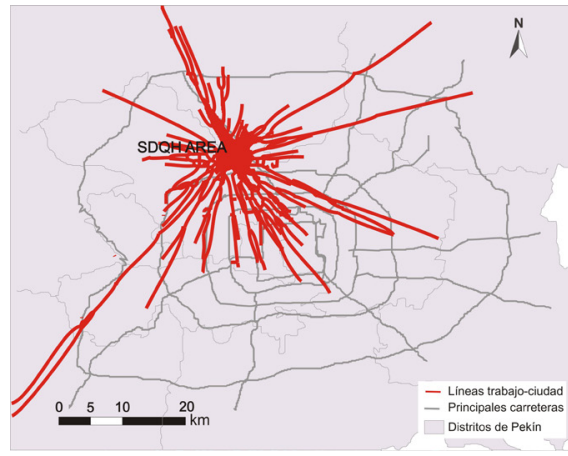

a) Desplazamientos al trabajo por parte de las personas encuestadas.

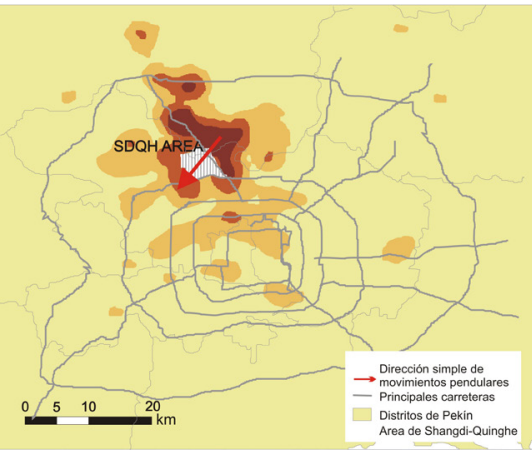

b) Lugar de alojamiento y dirección general de sus desplazamientos.

Fuente: elaboración propia a partir de la encuesta realizada.

parte de ellos tienen su lugar de empleo dentro de la cuarta CC, al norte de la avenida Changan y al noroeste de Shangdi-Qinghe. Por lo tanto, el sur, el sureste y el noroeste son las direcciones más destacadas de los viajes diarios al trabajo de los habitantes locales, pero la dirección general es hacia el sur. Los que trabajan y no residen en Shangdi-Qinghe, se mueven desde el este y el norte, debido a que una gran proporción habita en los barrios residenciales gigantes situados al norte de Pekín, como el de Huilongguan. Además, un 
volumen reducido de personas que viven entre la segunda y la cuarta CC acuden a su empleo hacia la zona periférica; así pues, la dirección del movimiento es principalmente del noreste y del sur a esta zona, y la dirección general es hacia el suroeste. En consecuencia, debido a la evidente deslocalización, en la hora punta de la mañana, grandes multitudes se mueven hacia el centro, y muchos trabajadores entran en esa zona desde el noreste y el sur, con lo que se causan graves atascos de tráfico en las carreteras.

\subsection{Actividades de ocio y compras}

Comparando los diarios, que registran las actividades de las personas analizadas durante una semana, con sus trayectorias registradas por el GPS, se pudieron extraer las referencias espaciales de cada actividad. El lugar de trabajo ya fijó la situación de los empleos con precisión. Basado en esto, el presente estudio se enfocó también hacia las dedicaciones no laborales analizando la densidad nuclear (figuras 8 a 10) de la distribución espacial de las actividades de ocio y compras de los tres grupos suburbanos.

Para los que viven y trabajan en Shangdi-Qinghe cuyos empleos están muy cercanos a sus casas, el espacio de su movimiento cotidiano está relativamente concentrado: hacen compras y otras actividades de ocio dentro de ShangdiQinghe, lo que demuestra que las instalaciones comerciales y de ocio de dicha zona ya pueden satisfacer las necesidades regulares de este grupo (figura 8). Las compras de los residentes locales que trabajan fuera se concentran dentro de Shangdi-Qinghe o en el centro de empleo Zhongguancun, aunque dicha actividad también se desarrolla en el CBD de la ciudad. La distribución de las actividades de ocio coincide con las de compras y se concentran alrededor de los lugares de residencia y de trabajo, pero están más dispersas con distribuciones bastante considerables por toda la zona noroeste entre la segunda y la

Figura 8. La distribución de las compras y el ocio de las personas que viven y trabajan en Shangdi-Qinghe

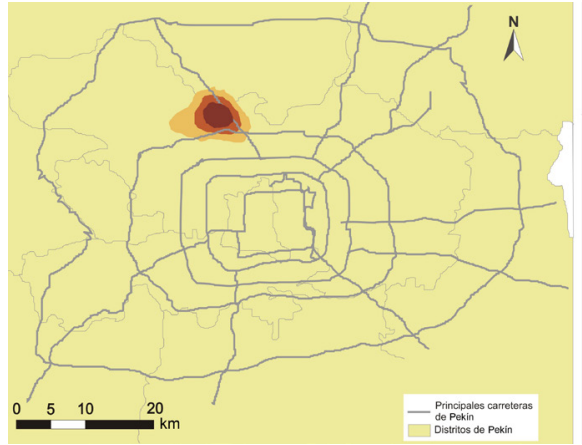

a) Compras.

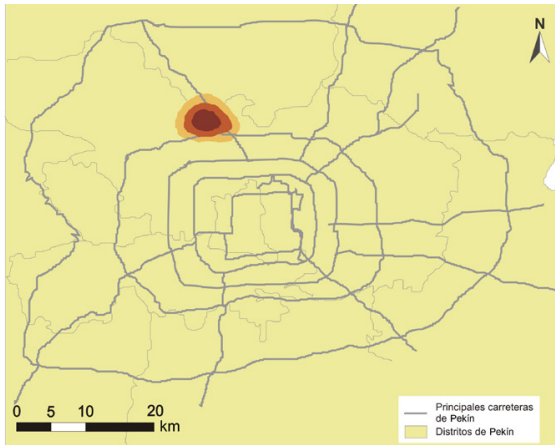

b) Ocio.

Fuente: elaboración propia a partir de la encuesta realizada. 
Figura 9. La distribución de las compras y los lugares de ocio de las personas que viven en Shangdi-Qinghe y trabajan fuera

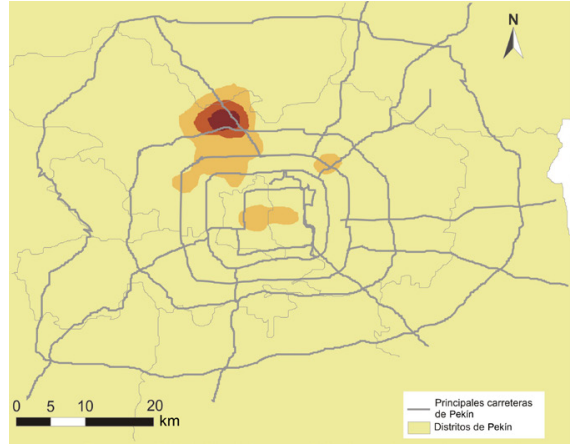

a) Compras.

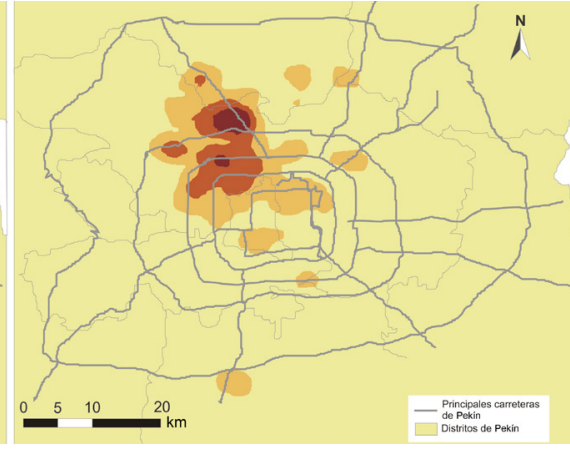

b) Ocio.

Fuente: elaboración propia a partir de la encuesta realizada.

Figura 10. La distribución de las compras y el ocio de las personas que trabajan en ShangdiQinghe y viven fuera

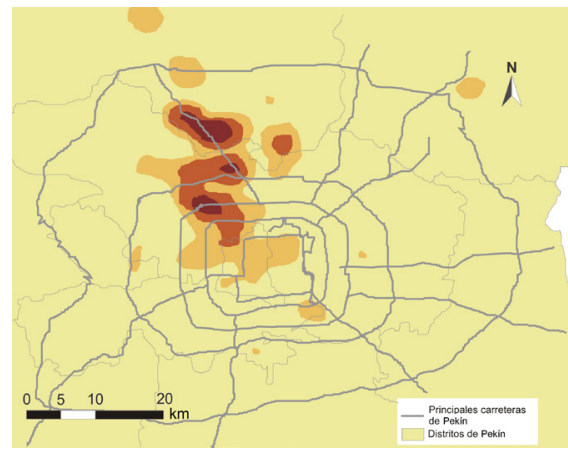

a) Compras.

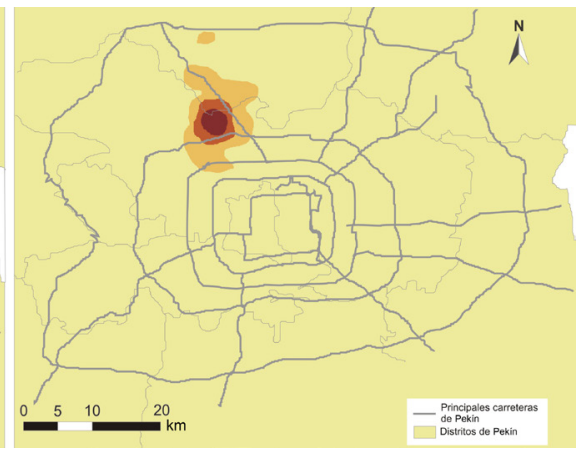

b) Ocio.

Fuente: elaboración propia a partir de la encuesta realizada.

sexta CC (figura 9). Los que trabajan en Shangdi-Qinghe y viven fuera hacen sus compras en diversos lugares: los más destacados hacia los que se dirige este grupo están entre la tercera y la sexta CC del noroeste, que incluye los centros comerciales en Huilongguan y Zhongguancun. Además, en Shangdi-Qinghe, su lugar de trabajo, y en el CBD de la ciudad también se encuentran algunas actividades de compras. No obstante, las de ocio están distribuidas mayoritariamente en el entorno donde tienen su empleo (figura 10).

A medida que las instalaciones comerciales y de ocio han aumentado continuamente en el suburbio durante estos años, las multitudes de residentes de 
dicha zona no tienen que entrar en el centro de la ciudad para realizar estas actividades de carácter cotidiano. En realidad, Shangdi-Qinghe, Zhongguancun y Huilongguan, al noroeste de Pekín, ya funcionan como subcentros comerciales y de ocio. Para los diferentes grupos de personas en Shangdi-Qinghe, las compras de los residentes y el ocio de los trabajadores se concentran en esta zona, pero el ocio de los residentes y las compras de los trabajadores se encuentran relativamente dispersos. Esto es, las compras conciernen prioritariamente al lugar de residencia, mientras que el ocio se corresponde con el lugar de trabajo.

\section{Conclusión}

En el rápido proceso de suburbanización de las ciudades chinas, las variedades del entorno, de los tipos de vivienda y de las comunidades residentes en las diferentes zonas destacan cada día más, lo cual exige una mayor cantidad de estudios geográficos y urbanos. Asimismo, las formas de organizar el espacio metropolitano han cambiado a raíz de la suburbanización y de la distancia existente entre los lugares de habitanza y de empleo, lo que ha provocado problemas como atascos de tráfico, injusticia social y reducción de la calidad de vida. Por ello, en los estudios sobre las zonas suburbanas, es necesario prestar mayor atención a los individuos que actúan en este espacio.

Tomando Pekín como ejemplo y partiendo del punto de vista espacial, esta investigación ha descrito el proceso de expansión de la ciudad en las últimas décadas, así como la plasmación espacial en los suburbios de categorías como habitantes, viviendas, industrias e instalaciones comerciales. También, desde el punto de vista humano, centrándose en el caso de Shandi-Qinghe de Pekín y basándose en los diarios sobre las actividades de los habitantes y los datos captados por GPS durante una semana, se ha estudiado en profundidad el espacio suburbano mediante el análisis de los lugares de compras y ocio, así como los trayectos hacia el trabajo de diferentes grupos de personas.

La investigación muestra que la población suburbana de Pekín ha aumentado continuamente, con abundantes barrios nuevos construidos durante los últimos años. De hecho, este espacio residencial se caracteriza por la segregación a nivel micro y la mezcla a nivel macro. Los polígonos suburbanos han acogido a las fábricas trasladadas y a la industria de tecnología avanzada, y los centros comerciales proliferan con instalaciones minoristas muy mejoradas. La zona entre la cuarta y la sexta CC se ha convertido en el espacio con mayor rapidez de desarrollo en Pekín durante los últimos años.

Considerando el distrito de Shangdi-Qinghe como estudio de caso, se ha clasificado a las personas objeto de muestreo en tres grupos según su lugar de vivienda y trabajo. Descubrimos que no solo los perfiles socioeconómicos de esos tres grupos muestran contrastes evidentes, sino también la movilidad cotidiana. Los que viven y trabajan en la misma zona no suelen tener permiso de conducir, ya que los espacios donde se desenvuelven sus actividades después del horario laboral se concentran alrededor de su casa. Buena parte de los que viven en Shangdi-Qinghe y trabajan fuera están registrados como ciudadanos 
pekineses, tienen mayores posibilidades económicas, están casados y son propietarios de sus viviendas. Teniendo la zona Zhongguancun al sur como su lugar de empleo principal, este grupo viaja hacia el centro para acudir al trabajo, y sus actividades de compras se concentran cerca de sus casas, mientras que las de ocio están distribuidas por distintos lugares. Entre los que desarrollan su activdad laboral en Shangdi-Qinghe y viven fuera de dicho distrito, a causa de la importancia de la industria local, la mayoría son jóvenes solteros con niveles educativos superiores, pero sin la ciudadanía pekinesa ni vivienda propia, y muchos de ellos viven al este y al norte de esa zona (Huilongguan es el barrio que más acoge). Las actividades de compras de este grupo están dispersas, pero las de ocio se concentran alrededor de su lugar de empleo. Así, podemos deducir que los habitantes del suburbio de Pekín se inclinan a hacer las compras cerca de su casa y a realizar las actividades de ocio allí donde trabajan.

Tras dos décadas, las zonas suburbanas de Pekín evolucionan poco a poco hacia la madurez. Con diferentes tipos de elementos reuniéndose en el suburbio, destaca, cada vez más, la diversidad de estos espacios. En relación con los entornos materiales, el uso del suelo se ha fragmentado, y las instalaciones no están distribuidas de forma equilibrada. Con respecto a las viviendas, tanto las privadas como las asequibles, las de las unidades de trabajo y las de reasentamiento están próximas unas de otras, pero se presentan segregadas. En lo referente a los comportamientos, existen distinciones entre diferentes grupos. $\mathrm{Al}$ mismo tiempo, el espacio suburbano avanza hacia su madurez: aparte de los habitantes, los trabajadores constituyen un colectivo para quienes dicho espacio forma parte ya de su vida cotidiana, pues las actividades de compras y de ocio se realizan dentro del mismo. El espacio suburbano de Pekín se está consolidando progresivamente, en consecuencia, la dependencia de la gran población suburbana con relación a la ciudad central disminuirá progresivamente, con los nuevos subcentros que concentran múltiples funciones y emergen en distintos lugares de la periferia.

\section{Referencias bibliográficas}

Chai, Yanwei y TA, Na (2009). «El desarrollo de los últimos 60 años y las perspectivas de los espacios urbanos de Pekín». Geografía Económica 9, 1421-1427. Edición original en chino.

Chen, Yelong y Zhang, Jingqiu (2010). «Un análisis de la influencia regional de las actividades laborales: El caso de Yizhuang, Pekín». Revista Académica de la Universidad Normal de la Capital (edición de las ciencias naturales), 6, 69-73. Edición original en chino.

FANG, Xiuqi et al. (2002). «Estudio sobre la expansión espacial y la zona de transición de Pekín en el último siglo». Urbanismo, 4, 56-60. Edición original en chino.

FEnG, Jian et al. (2004). «Las tendencias más recientes del desarrollo del suburbio de Pekín de los años 90 y sus soluciones». Urbanismo, 3, 13-29. Edición original en chino.

Gu, Zhaolin (1999). «El mecanismo del cambio de uso de la tierra de Pekín». Revista de Recursos Naturales, 4, 307-312. Edición original en chino. 
Huang, Youqin (2005). «From Work-unit Compounds to Gated Communities: Housing Inequality and Residential Segregation in Transitional Beijing [M]». En: LAURence, J.C. y Ma, Fulong Wu (eds.). Restructuring the Chinese Cities: Changing Society, Economy and Space. Londres y Nueva York: Routledge, 192-221.

LI, Wei et al. (2008). "El desarrollo típico chino de las "ciudades al margen": Un análisis de la transformación urbana a la estructura de diversos centros de Pekín y Shanghai». Urbanismo Internacional, 4, 2-6. Edición original en chino.

Long, Dao y ChaI, Yanwei (2006). «El uso de los centros comerciales suburbanos gigantes por los ciudadanos pekineses: El caso del centro comercial Recursos Dorados de Pekín». Geografía Humana, 5, 117-123. Edición original en chino.

MA, Jing et al. (2011). «El mecanismo de influencia sobre las emisiones de carbono del tráfico generado en los viajes de los habitantes pekineses». Revista de Geografía, 8, 1023-1032. Edición original en chino.

Ma, Qingyu y Zhang, Wenchang (2006). «La distribución de la suburbanización de Pekín y sus factores de influencia». Estudio Geográfico, 1, 121-188. Edición original en chino.

WeI, Lihua y YAN, Xiaopei (2006). «La "fragmentación desequilibrada” de los espacios sociales en la urbanización de las metrópolis: El caso de Guangzhou». Urbanismo, 5, 55-60 y 87. Edición original en chino.

Wu, Sheng (1989). «Logros, problemas y sugerencias: La revisión y la perspectiva de la construcción de viviendas en Pekín durante los últimos 40 años». Urbanismo, 5, 13-18. Edición original en chino.

YAnG, Zhensah; CaI, Jianming; OtTens, Henk F.L. y Sliuzas, Richard (2013). «Beijing». Cities [en línea], 31, 491-506.

<http://dx.doi.org/10.1016/j.cities.2011.07.007>

Zhang, Yan y ChaI, Yanwei (2011). «El estudio comparativo de los viajes al trabajo basado en las regiones residenciales». Estudio Geográfico, 5, 1327-1340. Edición original en chino.

Zheng, Guo y Qiu, Shike (2005). «El desarrollo de los polígonos y la reestructuración del espacio urbano en la época de transformación: El caso de Pekín». Investigación y Desarrollo sobre las Regiones, 6, 39-42. Edición original en chino.

ZhenG, Guo y Zhou, Yixing (2005). «Un estudio sobre la influencia de los polígonos económico-técnicos a la suburbanización de Pekín». Revista de Urbanismo, 6, 23-26 y 47. Edición original en chino.

Zhou, Yixing (1996). «Reflexiones sobre la suburbanización de Pekín». Ciencias Geográficas, 3, 7-15. Edición original en chino.

- (1999). «Desarrollar y orientar la suburbanización aprovechando al máximo los fundamentos». Urbanismo, 4, 13-17 y 64. Edición original en chino.

Zhou, Yixing y Logan, John R. (2008) "Growth on the Edge: The New Chinese Metropolis». En: Logan, J.R. (ed.). Urban China in Transition. Malden: Blackwell, 140-160.

Zhou, Yixing y Meng, Yanchun (1998). «La tendencia de suburbanización de las ciudades grandes chinas». Revista de Urbanismo, 3, 22-27 y 64. Edición original en chino. 